Www.jmscr.igmpublication.org

Impact Factor 5.84

Index Copernicus Value: 83.27

ISSN (e)-2347-176x ISSN (p) 2455-0450

crossref DOI: _https://dx.doi.org/10.18535/jmscr/v5i7.159

Journal Of Medical Science And Clinical Research

IGM Publication

An Official Publication of IGM Publication

\title{
Clinical Assessment of $0.5 \%$ Levobupivacaine with $0.5 \%$ Bupivacaine Administration in the Management of Spinal Anaesthesia
}

\begin{abstract}
Author
Dr Rajeev Kumar

Assistant Professor, Department of Anesthesia, Shri Ramkrishna Institute of Medical Sciences \& Sanaka Hospitals, Durgapur, WB, India

Corresponding Author

Dr Rajeev Kumar

Assistant Professor, Department of Anesthesia, Shri Ramkrishna Institute of Medical Sciences \& Sanaka Hospitals, Durgapur, WB, India

ABSTRACT

Spinal anaesthesia is frequently used for both optional and emergency caesarean section. Anesthesia-related mortality is decreased when general anesthesia is avoided. The study has planned with the aim to assess the clinical efficacy of Levobupivacaine compared with racemic Bupivacaine in spinal anesthesia for lower abdominal, pelvic and lower limb surgeries.

The total 50 enrolled patients were divided in 2 groups. Group A included the 50 patients received $3 \mathrm{ml}$ of $0.5 \%$ Levobupivacaine (15mg) $5 \mathrm{mg} / \mathrm{ml}$. Group B patients received $3 \mathrm{ml}$ of $0.5 \%$ Bupivacaine (15mg) $5 \mathrm{mg} / \mathrm{ml}$.

From the present study we conclude that, the time for onset of sensory and motor block showed no statistical significance in both groups. The duration of motor block was shorter, resulting in early ambulation in Levobupivacaine group. Haemodynamic parameters were stable in the Levobupivacaine group when compared with the Bupivacaine group. Fewer episodes of hypotension and bradycardia and other side effects were observed in Levobupavaine group.

Keywords: Spinal anaesthesia, Levobupivacaine, Bupivacaine, comparative study etc.
\end{abstract}

\section{INTRODUCTION}

Spinal anaesthesia (or spinal anesthesia), also called spinal block, subarachnoid block, intradural block and intrathecal block, ${ }^{[1]}$ is a form of regional anaesthesia involving the injection of a local anaesthetic into the subarachnoid space, generally through a fine needle, usually $9 \mathrm{~cm}$ (3.5 in) long. For obese patients longer needles are available $(12.7 \mathrm{~cm} \mathrm{/} 5$ inches). Spinal anaesthesia is a commonly used technique, either on its own or in combination with sedation or general anaesthesia. Examples of uses include:
- Orthopaedic surgery on the pelvis, hip, femur, knee, tibia, and ankle, including arthroplasty and joint replacement

- Vascular surgery on the legs

- Endovascular aortic aneurysm repair

- Hernia (inguinal or epigastric)

- Haemorrhoidectomy

- Nephrectomy and cystectomy in combination with general anaesthesia

- Transurethral resection of the prostate and transurethral resection of bladder tumours

- Hysterectomy in different techniques used 


\section{- Caesarean sections}

Spinal anaesthesia is the technique of choice for Caesarean section as it avoids a general anaesthetic and the risk of failed intubation (which is approximately 1 in 250 in pregnant women). It also means the mother is conscious and the partner is able to be present at the birth of the child. The post operative analgesia from intrathecal opioids in addition to non-steroidal anti-inflammatory drugs is also good.

If surgery allows, spinal anaesthesia is very useful in patients with severe respiratory disease such as COPD as it avoids intubation and ventilation. It may also be useful in patients where anatomical abnormalities may make tracheal intubation very difficult.

Regardless of the anaesthetic agent (drug) used, the desired effect is to block the transmission of afferent nerve signals from peripheral nociceptors. Sensory signals from the site are blocked, thereby eliminating pain. The degree of neuronal blockade depends on the amount and concentration of local anaesthetic used and the properties of the axon. Thin unmyelinated C-fibres associated with pain are blocked first, while thick, heavily myelinated A-alpha motor neurons are blocked moderately. Heavily myelinated, small preganglionic sympathetic fibers are blocked first. The desired result is total numbness of the area. A pressure sensation is permissible and often occurs due to incomplete blockade of the thicker A-beta mechanoreceptors. This allows surgical procedures to be performed with no painful sensation to the person undergoing the procedure. Some sedation is sometimes provided to help the patient relax and pass the time during the procedure, but with a successful spinal anaesthetic the surgery can be performed with the patient wide awake.

Levobupivacaineis a local anaesthetic drug belonging to the amino amide group. It is the Senantiomer of bupivacaine. ${ }^{[2]}$

Compared to bupivacaine, levobupivacaine is associated with less vasodilation and has a longer duration of action. It is approximately 13 percent less potent (by molarity) than racemic bupivacaine and has a longer motor block onset time. ${ }^{[3]}$

Bupivacaine is indicated for local infiltration, peripheral nerve block, sympathetic nerve block, and epidural and caudal blocks. It is sometimes used in combination with epinephrine to prevent systemic absorption and extend the duration of action. The $0.75 \%$ (most concentrated) formulation is used in retrobulbar block. ${ }^{[4]}$ It is the most commonly used local anesthetic in epidural anesthesia during labor, as well as in postoperative pain management. ${ }^{[5]}$

The study has planned with the aim to assess the clinical efficacy of Levobupivacaine compared with racemic Bupivacaine in spinal anesthesia for lower abdominal, pelvic and lower limb surgeries.

\section{METHODOLOGY}

The study is conducted in Shri Ramkrishna Institute of Medical Sciences \& Sanaka Hospitals, in Surgery department. The approval of ethical committee had been taken along with the consent from the patients were also taken. Total 50 patients having are group of 20-60 year were enrolled in to the study. The patients having other infections, Coagulopathy and bleeding disorders were excluded from the study.

The total 50 enrolled patients were divided in 2 groups. Group A included the 50 patients received $3 \mathrm{ml}$ of $0.5 \%$ Levobupivacaine $(15 \mathrm{mg}) 5 \mathrm{mg} / \mathrm{ml}$. Group B patients received $3 \mathrm{ml}$ of $0.5 \%$ Bupivacaine (15mg) 5mg/ml.

The following parameters were recorded in the both study group patients.

- Systolic blood pressure

- Diastolic blood pressure

- Mean arterial pressure

- Heart rate

- Time of Onset of Sensory Block

- Time of Onset of Motor Block

- Duration of sensory and motor block.

\section{RESULTS \& DISCUSSION}

The data from the two study groups were collected and presented as below. Total 50 
patient's data is presented in the study. The age group of the enrolled study group patients is ranging from age 20 to 60 years.

Table 1 : Age distribution of the Patients

\begin{tabular}{|l|c|c|}
\hline Age Group & Group A & Group B \\
\hline $20-30$ & 7 & 6 \\
\hline $31-40$ & 7 & 10 \\
\hline $41-50$ & 6 & 5 \\
\hline $51-60$ & 5 & 4 \\
\hline Total & 25 & 25 \\
\hline
\end{tabular}

Table 2 : Haemodynamic Parameters

\begin{tabular}{|l|c|c|}
\hline Parameter & Group A & Group B \\
\hline Systolic blood pressure mm of $\mathrm{Hg}$ & $115-130$ & $110-132$ \\
\hline Diastolic blood pressure $\mathrm{mm}$ of $\mathrm{Hg}$ & $71-80$ & $67-81$ \\
\hline Mean arterial pressure $\mathrm{mm}$ of $\mathrm{Hg}$ & $79-91$ & $74-88$ \\
\hline Heart rate & $67-88$ & $63-89$ \\
\hline Level of sensory block min & $7.6-10.2$ & $7.3-10.1$ \\
\hline Onset of Sensory level min & $4.2 \pm 1.3$ & $4.4 \pm 1.8$ \\
\hline Onset of motor blockade min & $6.2 \pm 1.6$ & $6.3 \pm 2.1$ \\
\hline
\end{tabular}

The table 2 indicates the Systolic blood pressure, Diastolic blood pressure, Mean arterial pressure, Heart rate, Level of sensory block, Onset of Sensory level and Onset of Sensory level. The Systolic blood pressure fall is found more in Bupivacaine group compared to Levobupivacaine group. There is no major difference in the Diastolic blood pressure. Levobupivacaine group shows more hemodynamic stability.

Table 3 : Frequency of Side Effects

\begin{tabular}{|l|c|c|}
\hline Parameter & Group A Patients & Group B Patients \\
\hline Hypotension & 1 & 5 \\
\hline Bradycardia & 2 & 4 \\
\hline Nausea/Vomiting & 1 & 2 \\
\hline Shivering & 1 & 1 \\
\hline
\end{tabular}

More cases of Hypotension and bradycardia were recorded in Bupivacaine group compared to Levobupivacaine group. Nausea and shivering are also the other side effects seen in the both study group.

Our study shows no difference between Levobupivacaine and Bupivacaine for the time of onset for sensory block. Guler et al ${ }^{[6]}$ conducted a study: Levobupivacaine versus Racemic Bupivacaine for Spinal Anaesthesia showed similar results. This is consistent with the findings in studies conducted by Casati et al [7], Mantouvalou et al ${ }^{[8]}$, Sathikarnmanee et al ${ }^{[9]}$ in which the time of onset of sensory block showed no difference.

Present study differs with Guler et al, Vanna et al ${ }^{[6]}$ in which the time of onset of motor block was faster in Bupivacine group than Levobupivacaine group.

In a study by $\mathrm{F}$ Fattorini and $\mathrm{Z}$ Ricci et. $\mathrm{Al}{ }^{[10]}$ there was no significant difference in the onset of motor block between Bupivacaine and Levobupivacaine group which coincides with the findings of our study. This is also supported by a study conducted by Erbay et al ${ }^{[11]}$, showing no statistical significant difference in the onset of motor block in Levobupivacaine and Bupivacaine group.

Bupivacaine remains the most widely used and cost effective, long acting local anaesthetic used in spinal anaesthesia. But, it comes with its own disadvantages like hypotension, bradycardia, cardiotoxicity and neurotoxicity. Levobupivacaine has a potentially greater margin of safety than the racemic Bupivacaine. The unbound fraction of Levobupivacine was significantly lower than that of unbound Bupivacaine because of its increased protein binding affinity. The early clinical presentation of toxicity in Levobupivacaine mostly consisted of central nervous system symptoms like drowsiness, disorientation, slurred speech which may complicate with tonic clonic seizures in some cases. These symptoms are generally self-limiting or respond to anti convulsive treatment. The susceptibility for seizure activity after intoxication with Levobupivacaine is 1.5 to 2.5 times less than that after racemic Bupivacaine.

\section{CONCLUSION}

From the present study we conclude that, the timefor onset of sensory and motor block showed nostatistical significance in both groups. The duration of motor block was shorter, resulting in early ambulation in Levobupivacaine group. Haemodynamic parameters were stable in the Levobupivacaine group when compared with the Bupivacaine group. Fewer episodes of 
hypotension and bradycardia and other side effects were observed in Levobupavaine group.

\section{REFERENCES}

1. Bronwen Jean Bryant; Kathleen Mary Knights (2011). Pharmacology for Health Professionals. Elsevier Australia. pp. 273-. ISBN 978-0-7295-3929-6.

2. Burlacu CL, Buggy DJ (April 2008). "Update on local anesthetics: focus on levobupivacaine". Ther Clin Risk Manag. 4 (2): 381-92. PMC 2504073 Freely accessible. PMID 18728849.

3. Gulec D (Apr 2014). "Intrathecal bupivacaine or levobupivacaine: which should be used for elderly patients?.". J Int Med Res. 42 (2): 376-385. PMID 24595149.

doi:10.1177/0300060513496737.

4. Lexicomp. "Bupivacaine (Lexi-Drugs)". Retrieved 20 April 2014.

5. Miller, Ronald D. (November 2, 2006). Basics of Anesthesia. Churchill Livingstone.

6. Guler, Gulen, et al. "A comparison of spinal anesthesiawith levobupivacaine and hyperbaricbupivacaine for cesarean sections: A randomized trial." Open Journal of Anesthesiology, Vol.2 No.3 (2012).

7. Andrea Casati, Elena Moizo, Chiara Marcheti et al,Prospective randomized doubleblindcomparison ofunilateral spinal anaesthesia with hyperbaric bupivacaine, ropivacaine \& levobupivacaine for inguinal herniorraphy. Anaes. Anal.; 2004;99;1384-92.

8. M Mantouvalou, S Ralli, H Arnaoutoglou, $G$ Tziris and G Papadopoulos. Spinal anesthesia: Comparison of plain Ropivacaine, bupivacaine and levobupivacaine for lower abdominal surgery. Acta Anaesth. Belg.,2008,59,65-71.

9. Sathitkarnmanee T1, Thongrong C, Tribuddharat S, BnMT, Bn KP, Bn RK.: Acomparison of spinal isobariclevo- bupivacaine and racemic bupivacaine for lower abdominal and lower extremity surgery; J Med Assoc, Thai. 2011Jun 94(6):716-20.

10. F Fattorini, Z Ricci et al; levobupivacaine versus racemicbupivacaine for spinal anaesthesia in major orthopaedicsurgery; Minerva Anestesiol 2006;72:637-44.

11. Hakan Erbay R, Ermumcu O, Hanci V, Atalay H.Acomparison of spinal anesthesia with low-dose hyperbariclevobupivacaine and hyperbaric bupivacaine for transurethral surgery:a randomized controlled trial. Minerva Anestesiol. 2010;76:992-100. 